\title{
Uma abordagem de design para MOOC: investigação da articulação do design instrucional \& de interação
}

\author{
Filipe A. Garrido, Beatriz B. do Rêgo, Rita Suzana P. Maciel, Ecivaldo de Souza Matos \\ ${ }^{1}$ Departamento de Ciência da Computação (DCC) \\ Instituto de Matemática e Estatística (IME) \\ Universidade Federal da Bahia (UFBA) \\ Salvador - BA - Brasil \\ \{filipe.garrido, beatrizbr, rita.suzana, ecivaldo\}@ufba.br
}

\begin{abstract}
MOOC is an acronym for Massive Open Online Course, an online education modality that offers free courses to diverse audiences with different ages, backgrounds and nationalities. It is believed that design approaches that integrate interaction design with instructional design may favor communicability in MOOC. In this sense, this article presents partial results of a Systematic Literature Mapping (SLM), whose objective was to identify studies and researches related to the processes of interaction design and instructional design applied to the MOOC. The results found point to the existence of research challenges of theoretical and methodological articulation between interaction design and instructional design in MOOC. The results presented in this article will guide a hybrid approach of design (articulated interaction design of instructional design) focused on MOOC.
\end{abstract}

Resumo. MOOC é um acrônimo para Massive Open Online Course, uma modalidade de educação online que oferta cursos gratuitos a diversos públicos, com diferentes idades, formações e nacionalidades. Acredita-se que abordagens de design que integrem design de interação com o design instrucional podem favorecer a comunicabilidade em MOOC. Nesse sentido, este artigo apresenta resultados parciais de um Mapeamento Sistemático de Literatura (MSL), cujo objetivo foi identificar estudos e pesquisas referentes a processos de design de interação e de design instrucional aplicados à MOOC. Os resultados encontrados apontam para a existência de desafios de pesquisa de articulação teórica e metodológica entre o design de interação e design instrucional em MOOC. Os resultados apresentados neste artigo guiarão uma abordagem híbrida de design (design de interação articulado ao design instrucional) focalizada em MOOC.

\section{Introdução}

O termo MOOC surgiu em 2008 por intermédio dos professores Dave Cormier da University of Prince Edward Island, e Bryan Alexander do National Institute for Technology in Liberal Education, sendo um acrônimo para Massive Open Online Course (Curso Online Massivo Aberto) [Grainger 2013]. Segundo João Mattar, MOOC é um tipo de curso online, aberto (gratuito e sem pré-requisitos para participação) e massivo (disponível a um grande número de alunos e com vasto material), podendo ser instanciado em diferentes tipos de plataformas web, para além dos tradicionais ambientes virtuais de aprendizagem, como o Moodle [Mattar 2013]. 
VII Congresso Brasileiro de Informática na Educação (CBIE 2018)

Anais do XXIX Simpósio Brasileiro de Informática na Educação (SBIE 2018)

O MOOC tem sido considerado uma das tendências mais proeminentes para o ensino superior dos últimos anos [Baturay 2015]. Esse fato se deve ao grande número de instituições de ensino superior que possuem plataformas próprias ou parceria com outras instituições para lançar cursos para o público externo ao campus [Matta e Figueiredo 2013, Boall et al. 2015, Dal Forno e Knoll 2013, Rodrigues et al. 2016b].

A pluralidade de perfis, necessidades e interesses de pessoas que procuram cursos em MOOC, aliada à quantidade massiva de acessos às plataformas de MOOC, representam um desafio de pesquisa para a comunidade epistêmica da Informática na Educação, especialmente na concepção de propostas de design de MOOC [Fassbinder et al. 2014], seja no aspecto de interação, sob a perspectiva do Design de Interação; seja no aspecto da abordagem pedagógica/andragógica, sob a perspectiva do Design Instrucional/Educacional.

Nesse sentido, este artigo apresenta resultados parciais de um Mapeamento Sistemático de Literatura (MSL), cujo objetivo foi identificar e analisar estudos e pesquisas referentes a processos de design de interação e de design instrucional aplicados à MOOC. Esses resultados já explicitam alguns problemas e algumas soluções, por meio de técnicas, métodos ou abordagens de design de interação ou design instrucional para MOOC.

O texto a seguir está estruturado em mais quatro seções, a citar: fundamentação teórica; a metodologia aplicada na condução do MSL, os resultados parciais encontrados até o momento e, por fim, as considerações parciais e etapas futuras de pesquisa.

\section{Fundamentação teórica}

O projeto de design de sistemas computacionais interativos e o planejamento das interações dos usuários sobre estes sistemas é tema de estudo do Design de Interação (DI) [Rogers et al. 2013]. Todavia, para Lowgren, o DI não é necessariamente uma área, mas o processo de modelagem das artefatos digitais para o uso humano [Löwgren 2013]. Neste artigo, consideramos a definição de Lowgren por favorecer a relação conceitual entre o DI e o design instrucional.

Segundo Filatro, o Design Instrucional (DInst) é um processo de concepção cur$\operatorname{ricular}^{1}$ em qualquer nível ou modalidade educacional, com o objetivo de produzir resultados de aprendizagem específicos; baseados não apenas na pesquisa pedagógica, mas também em práticas educacionais contemporâneas [Filatro e Piconez 2004].

Unificar esforços dessas duas áreas, DI e DInst, na concepção de MOOC pode favorecer a redução de custos de manutenção de cursos, a integração dos discurso de metacomunicação de interface, reduzir ruídos de comunicação entre os membros da equipe técnico-pedagógica de concepção dos cursos, além de agilizar processos ora realizados separadamente.

Uma abordagem híbrida de design (design de interação articulado com design instrucional) focalizada na comunicação usuário-sistema e usuário-sistema-usuário, mitiga as rupturas de comunicabilidade e favorece a construção colaborativa de conhecimento [Garrido 2018].

\footnotetext{
${ }^{1}$ Inventário de atividades implementadas com vistas a conceber, organizar e planejar uma ação educacional ou de formação, incluindo a definição de objetivos, conteúdos, métodos (incluindo avaliação) e materiais de aprendizagem, bem como disposições para a formação de professores e formadores (CEDEFOP - European Centre for the Development of Vocational Training, 2011)
} 
VII Congresso Brasileiro de Informática na Educação (CBIE 2018)

Anais do XXIX Simpósio Brasileiro de Informática na Educação (SBIE 2018)

\subsection{Trabalhos correlatos}

Foram encontrados quatro trabalhos correlatos, ou seja, outros mapeamentos/revisões relacionados ao design de interação ou design instrucional em MOOC, identificados durante o MSL: [Rodrigues et al. 2016a], [Sa'don et al. 2014], [Zawacki-Richter e Naidu 2016] e [Rocha e Fantinato 2013].

Em [Rodrigues et al. 2016a], Rodrigues, Brandão e Brandão orientaram sua revisão sistemática de modo semelhante à metodologia adotada neste estudo. Contudo, essa revisão foi conduzida de forma iterativa e selecionou bases diferentes das utilizadas neste estudo, IEEE Xplore, Google Scholar, Computers \& Education (periódico) e as publicações do Comissão Especial de Informática e Educação da Sociedade Brasileira de Computação (CEIE/SBC). Foram considerados artigos escritos na língua inglesa e portuguesa.

O objetivo da revisão sistemática de [Rodrigues et al. 2016a] foi identificar as causas da alta desistência no ensino a distância em estudos publicados entre 2008 e 2015. Nela, os autores buscaram confirmar se a proposta de um modelo para colaboração em pares para apoiar atividades de ensino e aprendizagem na web poderia ser uma estratégia interessante para diminuir as altas taxas de abandono dos cursos online, inclusive MOOC.

Por sua vez, em [Sa'don et al. 2014], os autores apresentam uma revisão sistemática de evidências empíricas relacionadas ao MOOC em instituições de ensino superior (IES). Essa revisão fornece uma visão abrangente sobre as pesquisas de última geração realizadas no MOOC em IES, concentrando sua busca entre 2008 e 2014.

O terceiro estudo, diferentemente dos dois anteriores, é um mapeamento sistemático, cujo objetivo foi destacar as tendências de pesquisa em educação a distância nos 35 anos de publicações da Revista Educação a Distância ${ }^{2}$ [Zawacki-Richter e Naidu 2016]. Esse mapeamento identificou os MOOC como o principal tema de pesquisa da educação a distância no ano de 2014; afirmando a importância de se pesquisar e discutir sobre esse tema ainda atual.

O quarto estudo correlato encontrado utiliza de uma metodologia semelhante à abordada neste artigo. O artigo de [Rocha e Fantinato 2013] apresentou resultados de uma revisão sistemática, cujo objetivo foi identificar e avaliar abordagens de Software Product Line (SPL) para Business Process Management (BPM).

\section{Metodologia}

O mapeamento sistemático de literatura, cujos resultados preliminares serão apresentados nas seções seguintes, teve por objetivo:

(OE1) identificar abordagens/processos de Design de Interação para MOOC;

(OE2) identificar abordagens/processos de Design Instrucional para MOOC;

(OE3) identificar lacunas de pesquisa nos estudos encontrados.

A metodologia deste mapeamento se adequou às diretrizes propostas por [Kitchenham 2004]. Em conformidade com essas diretrizes foram seguidas as seguintes etapas: (i) planejamento e definição das questões de pesquisa; (ii) aplicação dos critérios de inclusão e exclusão; (iii) execução do mapeamento e (iv) sumarização dos resultados.

\footnotetext{
${ }^{2} \mathrm{https}: / / \mathrm{mc}$. manuscriptcentral.com/cdie
} 
VII Congresso Brasileiro de Informática na Educação (CBIE 2018)

Anais do XXIX Simpósio Brasileiro de Informática na Educação (SBIE 2018)

\subsection{Planejamento e questões de pesquisa}

O planejamento consolidou-se com a definição do protocolo de pesquisa para mitigar erros e orientar os pesquisadores durante a execução do mapeamento. No protocolo estão contidas as questões de pesquisa com suas hipóteses relacionadas.

Para atender aos objetivos definidos, foram elaboradas uma questão principal (QP0) e duas questões específicas (QP1 e QP2):

- QP0: Há abordagens, diretrizes ou modelos integrados de design instrucional e design de interação aplicados a MOOC?

Rationale: A QP0 foi definida com o propósito de investigar se existem estudos que colaborem com a proposta de design híbrido indicada por [Garrido 2018].

Hipótese: Existem estudos que proponham abordagens, diretrizes ou modelos integradores de design instrucional e design de interação aplicados a MOOC.

Hipótese negativa: Não existem estudos que proponham abordagens, diretrizes ou modelos aplicados a MOOC que integrem design instrucional e design de interação.

- QP1: Quais abordagens, diretrizes ou modelos de design de interação têm sido aplicados a MOOC?

Rationale: A QP1 foi definida com o propósito de identificar estudos que utilizem abordagens, diretrizes ou modelos de design de interação aplicadas a MOOC.

Hipótese: Existem estudos que utilizem abordagens, diretrizes, modelos de design de interação aplicados a MOOC.

- QP2: Quais abordagens, diretrizes ou modelos de design instrucional têm sido aplicados a MOOC?

Rationale: A QP2 foi definida com o propósito de identificar estudos que utilizem abordagens, diretrizes ou modelos de design instrucional aplicadas a MOOC.

Hipótese: Durante as buscas nas bases de dados para realização desse MSL serão encontrados estudos que utilizem abordagens, diretrizes ou modelos de design instrucional aplicados a MOOC.

\subsection{Critérios de Inclusão e de Exclusão}

Os trabalhos que não atenderam todos os critérios de inclusão ou ao menos um critério de exclusão foram retirados (excluídos) do MSL. Os demais estudos foram mantidos nas etapas subsequentes do mapeamento.

\section{Critérios de Inclusão}

CI1. Estudo cujo objeto de pesquisa estivesse diretamente relacionado a Massive Open Online Course.

CI2. Estudo que apresentasse prática ou processo de design de interação ou design instrucional.

\section{Critério de Exclusão}

CE1. Artigo duplicado.

CE2. Artigo de literatura cinza.

CE3. Artigo com menos de cinco (5) páginas (short paper). 
VII Congresso Brasileiro de Informática na Educação (CBIE 2018)

Anais do XXIX Simpósio Brasileiro de Informática na Educação (SBIE 2018)

CE4. Estudo secundário (revisão e mapeamento de literatura).

CE5. Artigo indisponível (de acesso restrito ou não encontrados).

CE6. Artigo com insuficiência de conteúdo para os objetivos deste mapeamento.

CE7. Artigo escrito em idioma diferente do inglês.

\subsection{Execução}

Para execução do MSL foram criados dois conjuntos de strings. Elas foram compostas pelo termo MOOC e Massive Open Online Course em conjunto com os demais termos referentes a Design de Interação (interaction design) e Design Instrucional (instructional design), como pode ser visualizado no Quadro 1 e no Quadro 2.

Quadro 1. MOOC e termos relacionados

\begin{tabular}{c}
\hline MOOC and Interaction Design \\
MOOC and Instructional Design \\
MOOC and Interaction Design and Instructional Design \\
MOOC and (Interaction Design or Instructional Design) \\
\hline
\end{tabular}

\section{Quadro 2. Massive Open Online Course e termos relacionados}

Massive Open Online Course and Interaction Design
Massive Open Online Course and Instructional Design
Massive Open Online Course and Interaction Design and Instructional Design
Massive Open Online Course and (Interaction Design or Instructional Design)

Foram considerados os estudos publicados até o mês de junho de 2018, cujas buscas ocorreram na seguintes bases, considerando a expressiva quantidade de estudos indexados e relevância destas para a área de Ciência da Computação: ACM Digital Library ${ }^{3}$, IEEE Xplorer, Scopus e Science Direct.

Para efeitos de acurácia, optou-se por utilizar as ferramentas de busca avançada oferecidas por cada base, com uso de filtros e adaptações das strings. A adaptação para busca em cada base supracitada pode ser conferida em http://bit.ly/2xmlOxa.

As buscas nas bases selecionadas retornaram 309 trabalhos (considerando os artigos duplicados). O gráfico da Figura 1 apresenta a quantidade de trabalhos encontrados em cada base, apresentando o percentual em relação ao total. Após retirar os artigos duplicados, restaram 215 trabalhos. Dando-se início as subetapas da execução do mapeamento ( $1^{\circ}$ filtro).

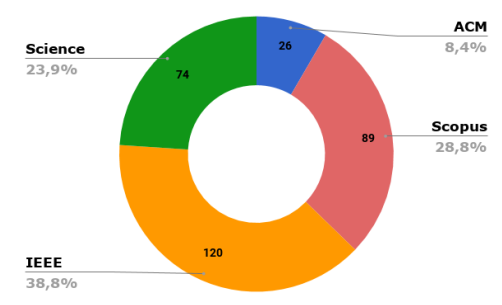

Figura 1. Quantidade de artigos encontrados em cada base - dados brutos

Nessa subetapa, após a aplicação dos critérios de exclusão e inclusão, foi realizada a leitura dos títulos, resumos e palavras-chave de cada artigo. Para isso, utilizou-se o Mendeley ${ }^{4}$ como ferramenta de suporte.

\footnotetext{
${ }^{3}$ https://dl.acm.org/

${ }^{4}$ https://www.mendeley.com/
} 
VII Congresso Brasileiro de Informática na Educação (CBIE 2018)

Anais do XXIX Simpósio Brasileiro de Informática na Educação (SBIE 2018)

Os artigos em que ocorreram divergências entre os dois pesquisadores quanto à adequação aos critérios de inclusão ou de exclusão, foram revisitados em uma subetapa de conciliação para reavaliação cruzada dos estudos divergentes. Nesse momento, um pesquisador apresentou os motivos de aceitação do estudo para o outro, caso houvesse consenso o estudo passaria para próxima fase. Caso contrário, seria descartado. Em seguida, esses pareces foram submetidos a dois pesquisadores mais experientes para validação.

Na segunda sub etapa da execução do mapeamento, realizou-se a leitura das seções de introdução e conclusão de todos os trabalhos aceitos na fase anterior, totalizando 179 artigos. Os artigos, cuja leitura da introdução e da conclusão não foram suficientes para o julgamento de aderência ao MSL, foram lidos na íntegra por meio das técnicas de leitura dinâmica, skimming e scanning, por não exigirem uma leitura precisa e detalhada do texto por completo.

Em casos que a divergência de opiniões se manteve, realizou-se nova rodada de reavaliação cruzada. Ao final dessa etapa restaram 43 artigos. Ressalta-se que todo o processo deste MSL foi realizado por 4 pesquisadores, dois estudantes em nível de mestrado e dois professores da pós-graduação em ciência da computação da UFBA.

Com o objetivo de estabelecer um ranking entre os artigos selecionados, considerando sua relevância para o alcance dos objetivos do MSL, foram aplicados os seguintes critérios de qualidade sobre os trabalhos selecionados até essa etapa:

- a estrutura é adequada e de fácil compreensão;

- os objetivos são claramente definidos;

- o problema de pesquisa é claramente definido;

- a metodologia de pesquisa é claramente descrita;

- uma prática de Design de Interação ou Instrucional aplicado a MOOC é apresentada;

- o processo de concepção ou avaliação de um MOOC é descrito;

- o processo de Design de Interação de um MOOC é descrito;

- o processo de Design Instrucional de um MOOC é descrito;

- a avaliação do Design de Interação ou Instrucional do MOOC é apresentada; e

- um guideline ou similar com melhores práticas para concepção de MOOC é apresentado.

Para cada critério de qualidade atribuiu-se uma das seguintes notas: zero (0), meio (0.5) ou um (1). Essas notas foram decorrentes da avaliação dos pesquisadores quanto ao nível de cumprimento do critério de qualidade. Os valores numéricos representam Não (0), Parcial (0.5) e Sim (1.0).

Estipulou-se nota mínima de seis $(6,0)$ para que o artigo fosse considerado selecionado à fase de extração de dados. Portanto, após aplicar os critérios de qualidade, os estudos que obtiverem nota igual ou maior que seis $(6,0)$, mediante a soma das notas atribuídas por critério na classificação, foram selecionados para a fase seguinte, extração de dados.

Na terceira subetapa foi realizado a extração de dados, os trabalhos foram lidos na íntegra para obtenção dos dados que atendessem aos objetivos do mapeamento sistemático, considerando os critérios de qualidades definidos pelos pesquisadores. 
VII Congresso Brasileiro de Informática na Educação (CBIE 2018)

Anais do XXIX Simpósio Brasileiro de Informática na Educação (SBIE 2018)

A Figura 2 destaca o número de artigos encontrados nas buscas iniciais, depois selecionados em cada subetapa da execução do MSL, após aplicação dos critérios de inclusão \& exclusão, e a quantidade final de artigos que passaram à leitura integral e aplicação dos critérios de qualidade.

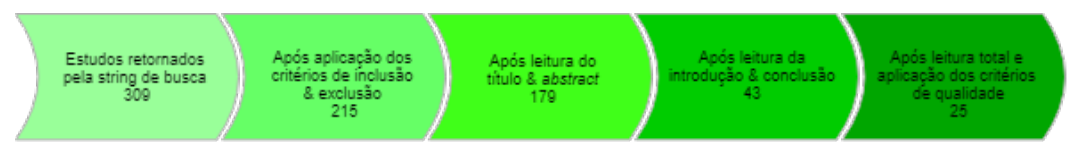

Figura 2. Quantidade de artigos selecionados na realização do MSL

\section{Resultados encontrados}

Os achados, 25 (vinte e cinco) artigos no total, foram agrupados como respostas aos objetivos deste MSL. Alguns trabalhos foram destacados em suas respectivas subseções com as contribuições julgadas relevantes pelos pesquisadores.

\subsection{Há abordagens, diretrizes ou modelos integrados de design instrucional e design de interação aplicados a MOOC?}

O MSL confirmou a hipótese nula de que não existem estudos que concebam/executem abordagens, diretrizes ou modelos integradores de design instrucional e design de interação aplicados a MOOC. Ao menos, não foram encontrados.

As buscas nas bases de dados selecionadas não retornaram trabalho que relacionassem (integrassem) design instrucional e de interação aplicado-os em MOOC; logo, não foram encontrados abordagens, diretrizes ou modelos com tal característica integrativa. Com isso, identificou-se uma lacuna de pesquisa.

Ressalta-se que o resultado do design instrucional é compreendido como parte integrante da interação do estudante com/no curso [Filatro 2008]. Entretanto, a ausência de estudos que relacionem esses campos de design, indica um desafio para a concepção de processo híbrido de design de MOOC.

\subsection{Quais abordagens, diretrizes ou modelos de design de interação têm sido aplicados a MOOC?}

O MSL confirmou a hipótese de que existem estudos que utilizam abordagens, diretrizes ou modelos de design de interação aplicados a MOOC. Foram identificados quatro (4) trabalhos que versam sobre essa questão.

Um dos estudos indica a dificuldade de tornar os MOOC tecnologicamente acessíveis a estudantes com deficiência, especialmente deficiência visual [Ramírez-Vega et al. 2017]. Os autores realizaram ensaios de acessibilidade nas páginas de cada curso, utilizando técnicas manuais e automáticas de avaliação. Em suas conclusões, os autores apresentam diretrizes gerais de acessibilidade para cursos e plataformas de MOOC, especialmente para as últimas que apresentam os maiores problemas nesse aspecto. Eles também evidenciam a necessidade de revisão dos processos de design instrucional seguidos pelas universidades provedoras de conteúdo. A ausência de informações mais precisas sobre como os conteudistas querem apresentar as instruções no conteúdo dificulta a criação de legendas ou representação das descrições e transcrições em áudio. Barreiras para o acesso universal ao curso oferecido. 
VII Congresso Brasileiro de Informática na Educação (CBIE 2018)

Anais do XXIX Simpósio Brasileiro de Informática na Educação (SBIE 2018)

Outro trabalho destacado é o estudo que propôs uma abordagem de design centrado no usuário utilizando um sistema de recomendação [Santos et al. 2014]. Essa adaptação baseou-se nas respostas de usuários durante seu levantamento de perfil em um ambiente de e-learning. Para avaliar o design instrucional do ambiente, os autores utilizam o feedback dos usuários como validador.

Dos artigos selecionados na etapa de extração, quatro (4) tratam prioritariamente sobre design de interação aplicado a MOOC. Entretanto, nenhum deles apresentou resultados sobre a qualidade da metacomunicação (comunicabilidade do sistema), focalizando apenas em aspectos como usabilidade e acessibilidade.

\subsection{Quais abordagens, diretrizes ou modelos de design de instrucional têm sido aplicados a MOOC?}

Vinte e um (21) trabalhos contribuíram na resposta à essa questão; em maior quantidade quando comparado ao design de interação (questão de pesquisa anterior). Esses trabalhos apresentaram, portanto, propostas, abordagens ou modelos de design instrucional aplicados a MOOC. Isso confirmou a hipótese de haver estudos que utilizam abordagens, diretrizes ou modelos de design instrucional aplicados a MOOC.

Uma evidência relevante apontada por um dos estudos é que a utilização de modelos para criação do Design Instrucional de um MOOC influencia a qualidade de aprendizado, como também influencia a taxa de abandono de seus estudantes [Delavar 2017].

Delavar investigou as características únicas dos MOOC, focalizando nas evidências do design instrucional; oferecendo um modelo para o design instrucional com resultados de eficácia da aprendizagem e medição da motivação dos estudantes utilizando parte do modelo ADDIE [Delavar 2017].

Outro estudo enfatizou que o design instrucional deve incorporar uma variedade de estratégias para chamar a atenção e interesse dos estudantes, de modo a estimular a curiosidade na investigação. Para isso, os autores utilizaram o modelo de design instrucional Attention, Relevance, Confidence and Satisfaction ${ }^{5}$ (ARCS) para alcançar o objetivo de engajar os estudantes em um curso MOOC [Huang e Hew 2017].

\subsection{Sumarização dos resultados}

Os artigos identificados neste MSL, ainda que tratem de áreas distintas, DI e DInst, convergem em diversos aspectos. Pois algumas ferramentas propostas têm papel fundamental na interação estudante-sistema-estudante, como o instrumento para ajuda por pares, Quick Helper MOOC [Howley et al. ]. Entretanto, os autores relatam seu impacto somente no aspecto instrucional, sem relacionar os seus reflexos na qualidade da interação dos e entre os estudantes.

Outros trabalhos encontrados propõem integrar ferramentas computacionais em MOOC, como [Santos et al. 2014]. Entretanto, a análise sobre o impacto dessa integração é parcial, ou sob a ótica da interação ou sob a ótica da instrução.

Qualquer artefato computacional inserido no ambiente MOOC poderá impactar no percurso educacional e na interação dos estudantes-usuários, como percebido nesse MSL. Sendo necessário analisar sua inserção de forma integrada desde o planejamento do

\footnotetext{
${ }^{5}$ Atenção, Relevância, Confiança e Satisfação. (Grifo nosso)
} 
VII Congresso Brasileiro de Informática na Educação (CBIE 2018)

Anais do XXIX Simpósio Brasileiro de Informática na Educação (SBIE 2018)

artefato. Entretanto, os trabalhos investigados neste MSL são parciais em suas propostas ou práticas, indicando atuação restrita a um das áreas, design de interação ou design instrucional.

\section{Considerações Parciais e Etapas Futuras}

Neste artigo foram apresentados os resultados parciais de um Mapeamento Sistemático de Literatura (MSL), cujo objetivo foi identificar estudos e pesquisas referentes a processos de design de interação e de design instrucional aplicados à MOOC.

Diante dos resultados apresentados neste artigo, constatou-se a existência de uma lacuna de pesquisa a ser explorada: o design integrado (interação e instrucional) para MOOC.

Portanto, são necessários estudos que permitam a articulação teórica e metodológica entre design de interação e design instrucional em MOOC. Nesse sentido, como trabalhos futuros endereçamos a conclusão desse MSL para formalizar a base de trabalhos que versem sobre design de interação ou design instrucional para MOOC e a concepção de uma proposta de design híbrido (design de interação articulado ao design instrucional) aplicado à MOOC, considerando aspectos de qualidade da interação pedagógica/andragógica e de comunicabilidade do sistema.

\section{Agradecimentos}

Agradecemos aos membros do Grupo de Pesquisa e Extensão em Informática, Educação e Sociedade - Onda Digital e à Fundação de Amparo à Pesquisa do Estado da Bahia (FAPESB) pelo financiamento desta pesquisa.

\section{Referências}

Baturay, M. H. (2015). An overview of the world of moocs. Procedia-Social and Behavioral Sciences, 174:427-433.

Boall, H., Chaves, M., e Stallivieri, L. (2015). Os moocs e o processo de internacionalização das instituições de ensino superior. XV Colóquio Internacional de Gestão Universitária - CIGU INPEAU/Universidade Federal de Santa Catarina, page [s. n.].

Dal Forno, J. P. e Knoll, G. F. (2013). Os moocs no mundo: um levantamento de cursos online abertos massivos. Nuances: estudos sobre Educação, 24(3):178-194.

Delavar, A. (2017). The effect of moocs instructional design model-based on student's learning and motivation. Man In India, 97(11):115-126.

Fassbinder, A., Delamaro, M. E., e Barbosa, E. F. (2014). Construção e uso de moocs: uma revisão sistemática. Brazilian Symposium on Computers in Education (Simpósio Brasileiro de Informática na Educação-SBIE), 25(1):332.

Filatro, A. (2008). Design instrucional na prática. Pearson Education do Brasil. São Paulo.

Filatro, A. e Piconez, S. C. B. (2004). Design instrucional contextualizado. São Paulo: Senac.

Garrido, F. A. (2018). Uma Abordagem Unificada de Design para Modelagem da Comunicação e Instrução para MOOC. Master's thesis, Mestrado em Ciência da Computação. Universidade Federal da Bahia - UFBA. 
VII Congresso Brasileiro de Informática na Educação (CBIE 2018)

Anais do XXIX Simpósio Brasileiro de Informática na Educação (SBIE 2018)

Grainger, B. (2013). Introduction to MOOCs: avalanche, illusion or augmentation. Policy Brief - UNESCO (july). Institute for Information Technologies in Education. URL:http://iite.unesco.org/pics/publications/en/files/3214722.pdf.

Howley, I., Tomar, G. S., Ferschke, O., e Rose, C. P. Reputation systems impact on help seeking in mooc discussion forums. IEEE Transactions on Learning Technologies, (1):1-14.

Huang, B. e Hew, K. F. (2017). Factors influencing learning and factors influencing persistence: A mixed-method study of mooc learners' motivation. Proceedings of the 2017 International Conference on Information System and Data Mining, pages 103110 .

Kitchenham, B. (2004). Procedures for performing systematic reviews. Keele, UK, Keele University, 33:1-26.

Löwgren, J. (2013). Interaction design-brief intro. The Encyclopedia of HumanComputer Interac tion, 2nd Ed.”. Aarhus, Denmark: The Interaction Design Foundation. http://www. interactiondesign. org/encyclopedia/interaction_design. html.

Matta, C. E. e Figueiredo, A. P. S. (2013). Mooc: transformação das práticas de aprendizagem. X Congresso Brasileiro de Ensino Superior a Distância, 10:1-15.

Mattar, J. (2013). Fóruns de discussão em educação à distância: Moodle, facebook e redu. Tecnologia Educacional, Revista Da Associação Brasileira De Tecnologia Educacional, 202:6-17.

Ramírez-Vega, A., Iniesto, F., e Rodrigo, C. (2017). Raising awareness of the accessibility challenges in mathematics moocs. Proceedings of the 5th International Conference on Technological Ecosystems for Enhancing Multiculturality, page 92.

Rocha, R. d. S. e Fantinato, M. (2013). The use of software product lines for business process management: A systematic literature review. Information and Software Technology, 55(8):1355-1373.

Rodrigues, P. A., Brandão, L. d. O., e Brandão, A. A. F. (2016a). Let us learn together!: Do complementary abilities foster pair collaboration in web-based learning? Frontiers in Education Conference (FIE), 2016 IEEE, pages 1-8.

Rodrigues, R. L., Ramos, J. L. C., Silva, J. C. S., e Gomes, A. S. (2016b). Discovery engagement patterns moocs through cluster analysis. IEEE Latin America Transactions, 14(9):4129-4135.

Rogers, Y., Sharp, H., e Preece, J. (2013). Design de interação: além da interação humano-computador. Bookman.

Sa'don, N. F., Alias, R. A., e Ohshima, N. (2014). Nascent research trends in moocs in higher educational institutions: A systematic literature review. Web and Open Access to Learning (ICWOAL), 2014 International Conference on, pages 1-4.

Santos, O. C., Boticario, J. G., e Pérez-Marín, D. (2014). Extending web-based educational systems with personalised support through user centred designed recommendations along the e-learning life cycle. Science of Computer Programming, 88:92-109.

Zawacki-Richter, O. e Naidu, S. (2016). Mapping research trends from 35 years of publications in distance education. Distance Education, 37(3):245-269. 